\title{
Guest editorial: Special issue on science of friction
}

\author{
Yonggang MENG ${ }^{1}$, Valentin L. POPOV ${ }^{2}$, Yuanzhong HU ${ }^{1}$ \\ ${ }^{1}$ State Key Laboratory of Tribology, Tsinghua University, Beijing 100084, China \\ ${ }^{2}$ Technische Universität Berlin, Institute of Mechanics, 10623, Berlin \\ Received: 08 June 2015 \\ (C) The author(s) 2015. This article is published with open access at Springerlink.com
}

Friction causes wear and energy dissipation of all moving parts under various operating conditions and environments. Approximately one third of the world's primary energy consumption is attributed to friction. Friction, as a decisive factor of energy efficiency, attracts vital interest of scientists and engineers trying to understand its origin and develop means to control friction through predictive models. Therefore, understanding the fundamental origins and mechanisms of friction is one of the great challenges in tribology.

This Special Issue of Friction is intended to report recent research progress in the basic science of friction, covering not only the fundamental mechanisms of friction at the atomic-scale, including experiments and simulations but also its industrial applications. Review papers, research articles and a short communication are included to demonstrate the breadth, importance and timeliness of the subject and to anticipate future developments. Six papers by tribologists and scientists having expertise in material science, physics and chemistry have been invited to pursue these aims. The contributions include two review articles on the effect of vapor adsorption on the tribological behaviors of different classes of materials and on the boundary lubrication by adsorption film, one research paper on the effects of the rate and solvation on friction and adhesion hysteresis between polymer brushes attached to curved surfaces, one paper on the micro scale study of frictional properties of graphene in ultra high vacuum, one on energy dissipation of atomic-scale friction based on onedimensional Prandtl-Tomlinson model and one short communication on the research works of Coulomb and Amontons and generalized laws of friction.
The first paper by Alazizi, Barthel, Surdyka, Luo, and Kim offers a comprehensive review of the effect of environment on friction and wear behavior of different classes of materials and potential applications of these effects for engineering problems. Covered are the influences of water and organic vapors on the friction of a wide range of materials including metals, glasses, ceramics, polymers, and carbon materials. The effect of surface roughness on lubrication with adsorbed vapor is highlighted as an important aspect of engineered surfaces that can be lubricated with adsorbed vapors. Furthermore, the utilization of adsorbed vapor as lubricants for miniature devices and as precursor for in situ synthesis of polymeric lubricants is thoroughly discussed.

Zhang and Meng review the development in the field of boundary lubrication by an adsorbed film and highlight historical breakthroughs in uncovering the mysterious but extremely useful process of boundary lubrication. This review focuses on the formation of a boundary film, the effects of the boundary film on the adhesion of rough surfaces, the behavior of an adsorption film in boundary lubrication and the boundary lubrication at the nanoscale. Especially, the methods of control of boundary lubrication are discussed exhaustively for various systems.

Beer, Kenmoé and Müser investigate the effects of a solvent and the model geometry on dissipation in polymer-brush systems by comparing molecular dynamic simulations based on different solvation schemes. The methods, simulation results and the dissipation mechanism are clearly presented. The rate dependence of the energy loss during the collision of brush-bearing asperities is different for explicit 
and implicit solvent, and the non-Newtonian rate dependences differ noticeably between head-on and off-center asperity collisions. Finally, friction is dramatically reduced compared to an under-saturated miscible polymer-brush system when the two opposing brushes are made immiscible, irrespective of the sliding direction.

Marchetto, Feser and Dienwiebel firstly discuss the frictional properties of epitaxial graphene on $\mathrm{SiC}$ using a microtribometer in ultra-high vacuum conditions. A low friction coefficient below 0.05 is observed, showing the excellent properties of graphene as solid lubricant. The lowering of the friction coefficient is explained by a reduction of the water layer on the sample and on the counter body. Moreover, the improved stability of the single-layer graphene in vacuum sheds light on the wear mechanism in ambient conditions. The anisotropy of the friction on $\mathrm{SiC}$ is also presented in detail and this effect is related to the presence of terrace steps on the surface of $\mathrm{SiC}(0001)$, while no anisotropy is found during the tests performed on $\mathrm{SiC}$ covered with graphene since the terrace steps are masked by the presence of graphene, which is explained with a "carpet behavior" of graphene.
Energy dissipation of atomic-scale friction is reported by Wang, $\mathrm{Ma}, \mathrm{Hu}, \mathrm{Xu}$ and Wang. By employing the Prandtl-Tomlinson model and solving the Langevin equation, a quantitative law of energy dissipation in sliding friction and stick-slip motion is clearly revealed for the first time. The energy dissipation ratio (EDR) is proposed to describe the energy reversibility during atomic-scale stick-slip, and it has been proven that the EDR is able to change continuously from $100 \%$ to 0 , covering stick-slip, intermediate and smooth sliding regimes. These theoretical investigations are valuable for understanding the atomistic mechanism of friction and shed lights on many novel tribological phenomena such as superlubricity and thermolubricity.

The closing paper by Popova and Popov sketches the contributions of Amontons and Coulomb to the science of friction from a historical and practical point of view in a short communication, including a short historical overview on Coulomb's works on friction, the main findings of Coulomb concerning dry friction, and the importance of the Coulomb's research for modern engineering and development of generalized laws of friction. Finally, some future study directions of the science of friction are highlighted. 